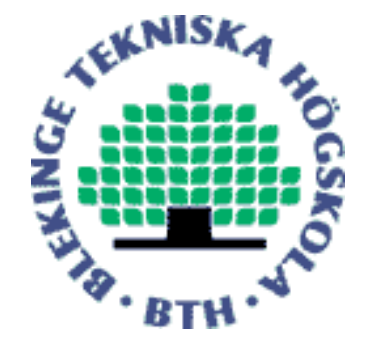

Copyright (C) 2012 IEEE.

Citation for the published paper:

A Simple Time-based Threshold Strategy for Dynamic Spectrum Access

Said Rutabayiro Ngoga, Yong Yao, Adrian Popescu

9th International Conference on Communications (COMM)

2012 Bucharest, Romania

This material is posted here with permission of the IEEE. Such permission of the IEEE does not in any way imply IEEE endorsement of any of BTH's products or services Internal or personal use of this material is permitted. However, permission to reprint/republish this material for advertising or promotional purposes or for creating new collective works for resale or redistribution must be obtained from the IEEE by sending a blank email message to pubs-permissions@ieee.org.

By choosing to view this document, you agree to all provisions of the copyright laws protecting it. 


\title{
A Simple Time-based Threshold Strategy for Dynamic Spectrum Access
}

\author{
Said Rutabayiro Ngoga, Yong Yao and Adrian Popescu \\ School of Computing, Blekinge Institute of Technology, 37179 Karlskrona, Sweden \\ E-mail : \{srn,yya,apo\}@bth.se
}

\begin{abstract}
In dynamic spectrum access networks, the unused licensed spectrum of primary users (PU) is opened to unlicensed secondary user (SU) for improving spectrum efficiency. The problem we study in this paper is to maximize the $\mathrm{SU}$ throughput while guaranteeing PU performance. We design a simple timebased threshold strategy to maximize the SU throughput while guaranteeing the PU performance by learning directly from the interaction with $\mathrm{PU}$ environment. At a given time, the $\mathrm{SU}$ decides if it should transmit and if so to protect the PU performance. It is observed that such strategies perform closely to the strategy optimized based on prior knowledge about PU traffic models. Simulations are used for performance evaluation.

Key words - Cognitive radio; dynamic spectrum access; timebased threshold strategies.
\end{abstract}

\section{INTRODUCTION}

We consider opportunistic spectrum access by secondary users (SUs) to the licensed spectrum of primary users (PUs). PUs have high priority in using the spectrum, oblivious to the existence of SUs. They can specify the acceptable loss behavior for their respective services or impose a unit cost per interruption on SUs access. On the other hand, SUs have low priority in using the spectrum. They are equipped with cognitive radio capability, and can only use spectrum opportunities left by PUs under protection requirement set a priori by PUs.

For the time being, the traditional "Listean-Before-Talk (LBT)" strategy has been extensively used to devise opportunistic spectrum access schemes [1], [2], [3]. This is motivated by the low infrastructure support and planning it requires. However, when used by an SU for transmission on an unslotted PU channel (or PU non-synchronized to it) cost automatically incurs.

In addition, the PU channel occupancy characterization consists of a stochastic model specific for PU's traffic. For example, it has been shown that a semi-Markov model can accurately characterize the channel occupancy specific for WLAN's packets transmission in [1]. Incorporating the statistical model into the LBT strategy gives the SU the ability to predict the PU environment's dynamics and behavior. It has been shown that this can significantly improve the SU performance, while the PU performance is guaranteed, in [2], [3].

However, real-world situation occurs when the statistical model for channel occupancy by PU are not detailed enough, or even not available. This gives space for new techniques that can achieve good performance by learning directly from interaction with the network environment, and without needing models of such environment.

In the paper, we report a simple time-based threshold strategy as a close-to-optimal transmission strategy that maximizes the SU throughput under explicit collision probability constraint. Specifically, we consider a system with one PU following a channel occupancy pattern model together with one SU. By learning through the interaction with PU environment, the SU uses its acquired knowledge to access PU channel. We introduce an approach for opportunistic spectrum access, and formally relate it to the traditional predictionlearning methods. As such, we assign costs by means of the difference between predicted and actual outcomes. Based on well known distribution transformations, we take the case of exponential distribution based methods and show that the SU can achieve close-to-optimal performance, yet without needing a predictive model of PU's channel occupancy.

The rest of the paper is organized as follow. Section II describes the system model that characterizes the PU and SU activities. Section III presents the structural properties of timebased threshold strategy. Section IV focuses on a special case of experiential distribution based method. Section V presents the simulation results. Section VI concludes the paper.

\section{SySTEM MOdEL}

We consider one spectrum band licensed to one PU. One SU is instantiated in form of opportunistic spectrum access (OSA) network. That is the spectrum opportunities left by the $\mathrm{PU}$ are available for SU under PU's protection requirement set by the PU a priori. The SU network can be viewed as an ad hoc link where the sender is synchronized to the receiver, and sensing and transmission share the same transceiver (antenna and RF front end) [4], [5].

\section{A. PU Model}

The traffic statistics of PU are such that the channel occupancy by PU evolves independently according to an alternating IDLE(0)-BUSY(1) process [1]. In particular, we denote the idle periods on the channel by a random variable $V$, the corresponding probability density function (PDF) and the cumulative distribution function (CDF) as $f_{V}(\cdot)$ and $F_{V}(\cdot)$, respectively. Associated with this is the mean idle period denoted $\rho_{V}$. Similarly, we also denote the busy periods on the channel by a random variable $U$ with mean $\rho_{U}$. The percentage of time the PU traffic is idle on the channel is therefore $\alpha=\rho_{V} /\left(\rho_{V}+\rho_{U}\right)$. 


\section{B. SU Model}

Like the PU, we assume the SU is also packetized, with both the packet length and slot length taking a duration $\delta$, namely a packet slot. We also assume that the SU packet slot is much less compared to the PU packet slot.

For the simplicity of analysis, we assume the SU performs perfect sensing on PU channel [5], [3], [2]. That is, the sensing period is negligible and the sensing outcome are error-free. Consequently, an SU can perfectly detect the beginning of the idle period on the PU channel, but cannot assure whether the PU will remain idle in the near future or not.

Besides, we assume that any small transmission overlap between PU and SU results in collision to both of them [2]. For simplicity, we consider a scenario in which collision corrupts the first PU packet slot for each new PU activity. Then, we define a collision instance as follows: a new PU activity starts on a particular channel while the SU has accessed the channel and has not yet finished its transmission.

\section{Stucture of an SU Channel Access Strategy}

First, we assume that the SU has always data to transmit. Hence, immediate spectrum access is always necessary. Based on this, each SU time slot consists of a sensing phase, followed by a transmission phase. By using its spectrum sensor on the PU channel, the SU senses whether the channel is idle or busy during the sensing phase. The sensing result is passed on to the spectrum access controller. If the channel is sensed busy after a sensing phase, no SU transmission takes place, and the SU will sense the channel again in the next time slot. But, if the channel is sensed idle after a sensing phase, the SU decides whether to transmit or not at all, according to some spectrum access strategy.

Besides, the spectrum access strategy should take into account the different sources of uncertainties that exist in the access process and their respective costs. In particular, if an SU transmits and the PU channel remains idle until the end of the transmission phase, an SU earns a unit reward for successful transmission. But, if the PU traffic resumes before the SU transmission phase ends, the SU incurs a unit cost for inducing a slot packet collision to the PU. Naturally, this yields a channel access strategy that can be implemented in a simpler threshold structure.

\section{Performance Measures}

An PU has a packet collision probability specified as $\eta$. It represents the maximum probability of collision allowed by the PU. The collision probability of an SU transmission strategy is expressed by the proportion of collided PU's packets in packets fully transmitted by the PU over a time interval $[0, T]$. If we assume that an PU's packet slot is of length 1 , the collision probability is denoted by $p_{c}$ and given by

$$
p_{c}=\lim _{T \rightarrow \infty} \frac{\text { Number of PU packets collision in } T}{\text { Number of PU packet transmitted in } T}
$$

The performance guarantee provided to PU for each PU's packet transmitted is thus

$$
p_{c} \leq \eta
$$

This is also the collision constraint imposed on SU transmissions. Normalized over the number of PU packet slots transmitted in each busy period of duration $\rho_{V}$, the PU collision constrain thus denotes $p=\eta \rho_{V}$. Next we define the SU throughput of a transmission strategy by

$$
J=\lim _{T \rightarrow \infty} \frac{\text { Number of SU access packet slots in } T}{\text { Number of available SU packet slots in } T}
$$

The goal of the transmission strategy is to maximize the SU throughput under the collision constraint.

\section{A Simple OSA AND PROPERTIES}

In this section we investigate on a class of threshold strategies for OSA build on the basis of learning from the interaction without requiring knowledge on the models of the environment's dynamics.

\section{A. Prelimiaries and Notations}

Consider a set of instances, $\left\{x_{i}\right\}_{i=1}^{n}$, with $x \in \mathcal{X}$. We are also given labels, $\left\{y_{i}\right\}_{i=1}^{n}$, with $y \in \mathbf{R}$. These instances and their labels are the set of $n$ training examples. For a new unlabeled example, we observe $x_{n+1}$ and we wish to predict the label $y_{n+1}$ by mean of some predictor $\phi(\cdot)$. This yields a problem for designing an on-line setting in which labels are predicted successively, each one being revealed before the next is predicted.

In our case, a spectrum access strategy consists of advanced signal processing techniques to capture spectrum opportunities, coupled with sophisticated networking protocols for nonintrusive opportunity exploitation. Therefore, each instance $x_{i}$ encodes the channel state information provided by the spectrum sensor on given channel in the spectrum. A random vector $\left\{x_{i}\right\}_{i=1}^{n}$ denotes the dynamics of a PU occupancy on a given channel. The labels, $\left\{y_{i}\right\}_{i=1}^{n}$, encode the idle dwell time as the observable gathered about the channel state.

\section{B. Problem Description}

Suppose we observe $z_{i}=\left(x_{i}, y_{i}\right)$ in sequence. After observing $z_{1}$ and $z_{2}$, we start predicting. For $n=3,4, \ldots$, just before observing $y_{n}$, we predict it based on what we have observed so far, $x_{n}$ and the previous instances $z_{1}, \ldots, z_{n-1}$.

Let $Z=z_{1} z_{2} \ldots$ denote independent and identically distributed random variables distributed according to an unknown probability distribution function $F$. The model construction rests on the assumption of the model of process generating the sequence $Z=z_{1}, z_{2}, \ldots$, i.e., the distribution assumption. We assume the distribution of the underlying random variables belongs to a parametric family. This family of distribution functions follows a pdf $f(\cdot \mid \theta)$, specified by an unknown parameter vector $\theta$.

Next, our concern is on how uncertainty about a future observation $y$ relates to the way we employ the model to decide whether to access a channel deemed idle or not. Among 
all plausible predictive models, we choose the model that predicts well, but also that has the advantage of having low operations cost. The problem reduces to find out a quantity of interest $\phi_{p}$, which interprets a predictor for the value taken by a future observation $y$ from the same underlying distribution. $p$ is a relative cost resulting from collision due to under-estimation of $y$.

Since $\phi_{p}$, like $\theta$, are unknown, we use a set of training examples as a sample $\left\{z_{1}, \ldots, z_{n}\right\}$ to estimate the quantity $\phi_{p}$ by $\hat{\phi}_{p}$. Then, the mapping

$$
\left(z_{1}, \ldots, z_{n}\right) \longrightarrow \hat{\phi}_{p}=\hat{\phi}_{p}\left(y_{1}, \ldots, y_{n}\right)
$$

allows for further decisions about whether to access the SU or not. The learning procedure will be expressed as a rule for updating $\hat{\phi}_{p}$. By extension, we call a possible value $d$ of $\phi_{p}$ a prediction.

\section{Cost Function}

So far, we have employed the estimated value $\hat{\phi}$ for the value taken by a future observation to make some decisions. In this respect, the loss or cost function is a certain function $C(y, d)$, which measures the cost incurred when a prediction $d$ is used for the value of a future observation $y$. Here the decision $d$ stands for the value we choose for the unknown quantity $y$.

Let us now define the cost function. For a preset collision constraint $p \in[0,1]$,

$$
C_{p}(y, d)=|y-d| \times\left[p \mathbf{1}_{\{y<d\}}+(1-p) \mathbf{1}_{\{y \geq d\}}\right]
$$

This means that $C_{p}(y, d)$ is the additional cost of the prediction $d$, and it is proportional to the absolute deviation from $y$ multiplied by a fraction depending on whether $d$ underestimates or over-estimates $y$. However, the collision cost occurring when $y<d$ is much more costly.

With the cost function in equation (4) as a specification for loss, the expected loss is

$E_{C_{p}(y, d)}=p \int_{-\infty}^{d}(y-d) f(y) d y-(1-p) \int_{d}^{\infty}(y-d) f(y) d y$

By differentiating with respect to $d$, we obtain the minimum of equation 5

$$
\frac{\partial}{\partial d} E_{C(y, d)}=-p \int_{-\infty}^{d} f(y) d y+(1-p) \int_{d}^{\infty} f(y) d y
$$

After equating to zero, we find the solution $d$ to equation 6 to be the $p$-th quantile of the output $y$, which denotes $\phi_{p}$.

\section{Prediction Intervals}

In the context of OSA, the SU transmissions are constrained to meet a collision profile set a priori by PU. Hereafter, we proceed with non-intrusive method of making predictions. In which case, we provide a threshold on the number of PU slot packets that can be lost as a result of colliding transmissions. Otherwise, too many collisions will affect the PU performance, which is unacceptable. A prediction interval, at a pre-specified level $p$, is an interval that we can compute by observing a realization of $z_{1}, \ldots, z_{n}$ such that, with probability $p$, the value of a future observation $y_{n}$ will be captured in this interval.

In this respect, let a set of $n$ training examples $z_{1}, \ldots, z_{n}$ denotes a random sample from a distribution belonging to a parametric family, admitting a pdf $f(\cdot \mid \theta)$, specified by an unknown parameter $\theta$. After we observe $x$, we want to predict $y$. Furthermore, let $p$ be the collision constraint and $\phi_{p}$ the $p$-th quantile assumed to be uniquely defined. Our objective is to construct a sample estimator $\hat{\phi}_{p}$ of the $p$-th quantile distribution, $\phi_{p}$, such that equation

$$
\operatorname{Pr}\left[y<\hat{\phi}_{p}\right]=p
$$

or

$$
E_{f}\left(\operatorname{Pr}\left[y<\hat{\phi}_{p}\right]\right)=p
$$

is satisfied.

Equation 7 indicates the exact prediction interval for a future unseen observation $y$, while equation 8 indicates the prediction interval for $y$ with zero coverage error.

\section{Familly of Exponetials Based Procedure}

Let a set of $n$ training examples $z_{1}, \ldots, z_{n}$ be such that the sequence $\left\{y_{i}\right\}_{i=1}^{n}$ denotes a positive exponential distribution random sample. The pdf and cdf are respectively given by $f(y)=\frac{1}{\theta} \exp \left(\frac{y}{\theta}\right)$ and $F(y)=1-\exp \left(-\frac{y}{\theta}\right)$. Let $z=(x, y)$ denote a new unlabeled example drawn according to $f$, and also let $p$ be the collision constraint. The optimal predictor $\phi_{p}$ satisfying (4) and (6), is the $p$-th quantile of the distribution, which is given by

$$
\phi_{p}=-\theta \ln (1-p)
$$

Proposition: It follows that if $\phi_{p}$ is estimated by

$$
\hat{\phi}_{p}=c_{n}(p) \omega
$$

where

$$
c(n)=\frac{1}{(1-p)^{\frac{1}{n}}}-1, \quad \omega=\sum_{n=1}^{n} y_{i}
$$

then, for a new unlabeled example, a zero coverage prediction interval

$$
E_{f}\left(\operatorname{Pr}\left[y<\hat{\phi}_{p}\right]\right)=p
$$

is satisfied [6].

\section{Simulation Results}

Simulation results are reported to illustrate the performance of the suggested threshold strategy using some specific parameters of the system. The selection of the parameter values is not crucial but representative of the general behavior of the system. 


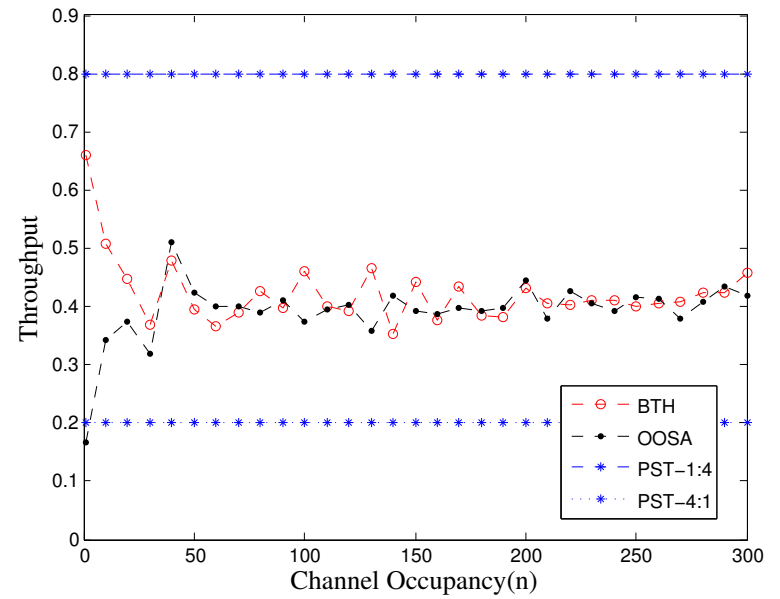

Figure 1. Comparison of the SU throughput with OOSA and PST. PST settings are $S=1, T=4$, and $S=4, T=1 . p=0.4$

\section{A. Simulation Setup}

The PU channel occupancy consists of $n$ consecutive idlebusy cycles, generated in continuous time. The length of each idle period $V$ is continuous with mean $\rho_{V}=40$, and follows the pdf $f_{V}(v)=\frac{1}{\rho_{V}} \exp \left(\frac{v}{\rho_{V}}\right)$. Whereas, the busy period $U$ is fixed to be $\rho_{U}=20$. Both the SU packet length and SU slot length are set to $\delta=0.1$.

\section{B. Performance of the Time Threshold Strategy}

We compare the performance achieved by the suggested time-based threshold strategy and that of the optimal opportunistic spectrum access (OOSA) suggested in [3]. In the case that the idle period is exponential distribution, OOSA allows a SU to initiate a transmission at the first time slot in which the channel is sensed idle and keep transmitting for a time $\phi_{p}$, where $\phi_{p}=-\rho_{V} \ln (1-p)$. Also, we compare the performance achieved by the suggested time-based threshold strategy with a periodic sense-transmit strategy (PST). The PST is an intuitive strategy operating periodically with two modes sleep mode and transmit mode of fixed durations, $S$ and $T$ respectively. In Fig. 1, for a normalized collision constraint fixed at $p$, we plot the maximum throughput achieved by the suggested strategy (called BTH) and both OOSA and PST. It is observed that the suggested time-based threshold strategy performs very close to the optimal performance achieved by OOSA, as the number of sample size increases. Whereas, the suggested strategy always obtains better or less performance than the PST, which indicates that the PST can not adapt to dynamic changes of the environments.

Next, we compare the number of collisions induced by the suggested strategy and both the OOSA strategy and the PST strategy. In Fig. 2 it is observed that the number of collisions induced by the suggested strategy is very close to that of OOSA. Whereas, the number of collisions induced by the suggested strategy is always higher or less compared to that of PST.

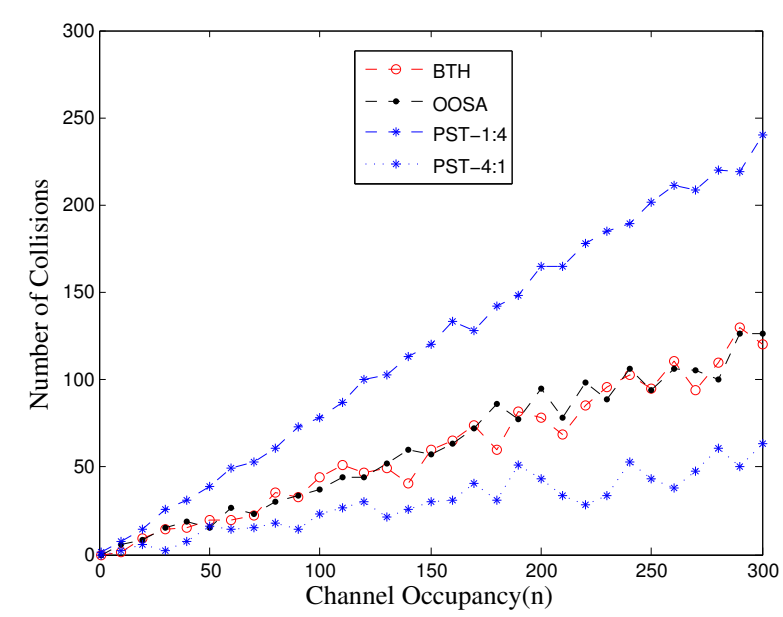

Figure 2. Comparison of number of collision induced by the SU with OOSA and PST. PST settings are $S=1, T=4$, and $S=4, T=1 . p=0.4$

\section{CONCLUSIONS}

We introduce a simple time-based threshold strategy as a close-to-optimal transmission strategy that maximizes the SU throughput under explicit collision probability constraint. Specifically, we consider a system with one PU following a channel occupancy pattern model together with one SU. By learning through the interaction with PU environment, the SU uses its acquired knowledge to access the PU channel. We introduce an approach for opportunistic spectrum access, and formally relate it to the traditional prediction-learning methods. As such, we assign costs by means of the difference between predicted and actual outcomes. Based on well known distribution transformations, we take the case of exponential distribution based methods and show that the SU can achieve close-to-optimal performance, yet without needing a predictive model of PU's channel occupancy. Future work is on the extension of the suggested method to non-parametric settings in which case the distributional functional form about the PU behavior may not be readily available.

\section{REFERENCES}

[1] S. Geirhofer, T. Lang, and B.M. Sadler, " Dynamic Spectrum Access in the Time Domain: Modeling and Exploiting White Space,' IEEE Comm. Mag., vol. 45, no. 5, May 2007.

[2] H. Senhua, L. Xin, and D. Zhi,“ Optimal Transmission Strategies for Dynamic Spectrum Access in Cognitive Radio Networks,”, IEEE Trans. Mob. Comp., vol. 8, no. 12, Dec. 2009

[3] X. Qinghai, L. Yunzhou, Z. Xiaofeng, X. Xibin, and W. Jing, “ A unified approach to optimal opportunistic spectrum access under collision probability constraint in cognitive radio systems, ” EURASIP J. Advances in Signal Processing - Special issue on advanced signal processing for cognitive radio networks 2010, Jan. 2010.

[4] Z. Qing, T. Lang, S. Q. Ananthram, and C. Yunxia, "Decentralized cognitive MAC for opportunistic spectrum access in ad hoc networks "IEEE J. Selected Areas in Comm., vol. 25, no. 3, Apr. 2007.

[5] Z. Qianchuan, S. Geirhofer, T. Lang, and B.M. Sadler, “ Opportunistic Spectrum Access via Periodic Channel Sensing "IEEE Trans. Signal Processing, vol. 26, no. 2, Jan. 2008.

[6] S. R. Ngoga and A. Popescu, "On time-based threshold stategies for Dynamic Spectrum Access " to be submitted. 